\title{
EI Trabajo Social Educativo: Perspectiva desde el Departamento de Consejería Estudiantil
}

Educational Social Work: Perspective from the Department of Student Counseling

Leonor Alexandra Rodríguez Álava, Dra. ${ }^{(1)}$

María José Loor Zamora. ${ }^{(2)}$

Ulbio Colón Durán Pico, MSc. ${ }^{(3)}$

(1), (2) y (3) Facultad de Ciencias Humanísticas y Sociales; Instituto de Ciencias Básicas Universidad Técnica de Manabí. Ecuador

Contacto: alexanroa32@hotmail.com

Receptado: 20/02/2018

Aceptado: 16/04/2018

\section{Resumen}

El área de acción del trabajador social es diversa; en el ámbito educativo, su trabajo se fortalece con otros profesionales, encontrándose con situaciones conflictivas relacionadas con estudiantes, familias, docentes y autoridades, que afectan el proceso formativo. El propósito de la investigación fue identificar la intervención del trabajador social como parte del equipo multidisciplinario del Departamento de Consejería Estudiantil (DECE) en el Distrito 4 del cantón Portoviejo - Manabí - Ecuador, zona 13D01. Para la puesta en marcha de este estudio cuali - cuantitativo se utilizó a nivel teórico los métodos inductivo - deductivo, análisis y síntesis, y la revisión bibliográfica que permitió fortalecer el sustento científico; a nivel empírico se aplicó encuesta a estudiantes, trabajadores sociales y directivos de las Unidades Educativas, el análisis estadístico para el procesamiento de datos. En los resultados se destaca que los problemas más comunes están relacionados con la disciplina y el rendimiento académico de los estudiantes; la función principal del trabajador social se circunscribe en la orientación a estudiantes y padres de familia, y la investigación; evidencia un rol protagónico y su aporte es valorado por los miembros de la comunidad educativa; de la misma manera se resalta la necesidad de emprender acciones de prevención que atenten al desarrollo armónico de los estudiantes y sus familias y la atención a los derechos de los grupos más vulnerables. 
Palabras clave: Enseñanza, asistencia social, agente de cambio, bienestar social.

\section{Sumary}

The action area of the social worker is diverse; In the educational field, his work is strengthened with other professionals, encountering conflictive situations related to students, families, teachers and authorities, which affect the training process. The purpose of the investigation was to identify the intervention of the social worker as part of the multidisciplinary team of the Department of Student Counseling (DECE) in District 4 of the canton of Portoviejo - Manabí Ecuador, zone 13D01. For the implementation of this qualitative study - quantitative at a theoretical level - the inductive methods - deductive, analysis and synthesis, and the bibliographical review that allows to improve the scientific sustenance; an empirical level was applied to students, social workers and managers of the Educational Units, the statistical analysis for data processing. The results highlight the most common problems related to the discipline and academic performance of students; the main function of the social worker is circumscribed in the orientation to students and parents, and the investigation; evidence a leading role and its contribution valued by members of the educational community; in the same way, it is due to the need to undertake prevention actions that threaten the harmonious development of students and families and the attention to the rights of the most vulnerable groups.

Keywords: Teaching, social assistance, agent of change, social welfare.

\section{Introducción}

El área de actuación de los profesionales en Trabajo Social a través del tiempo se ha circunscrito preferentemente en el sector social; razón por la que, de acuerdo al criterio de los autores de este trabajo, resulta necesario conocer un poco de su trayectoria hasta su inclusión en el ámbito educativo. Como profesión tuvo su origen en el siglo XIX en Estados Unidos e Inglaterra, como medio para ayudar a los pobres, los que eran vistos como un problema social, clasificados en diferentes categorías: las personas muy humildes que podían trabajar, los imposibilitados y personas que no tenían una actitud hacia el trabajo (Cárdenas, 2014 )

En Latinoamérica, como respuesta a los problemas sociales, políticos y culturales, surgen las escuelas de Servicio Social; por su parte, en Ecuador, este profesional nace para atender las necesidades básicas de los sectores populares y marginados. En las primeras décadas del siglo 
$\mathrm{XX}$, se desplegaron los centros de atención social, cuyos servicios fueron dirigidos a los niños y jóvenes, a la población adulta y a la familia, aspectos que confirman que la constitución profesional del Trabajo Social tiene sus raíces en el origen de las desigualdades sociales generadoras de la penuria y la opulencia; su objeto de estudio, se ubica en el espacio donde se generan las necesidades y a su vez las satisfacciones, (Frank, 2018).

En las últimas décadas, logró la ampliación del área de actuación descrita anteriormente; este profesional, a través del tiempo, ha diseñado y ejecutado políticas sociales de rehabilitación, protección, promoción y prevención, ejerciendo las funciones de investigador social, asesor, capacitador, organizador, programador, ejecutor, evaluador, gestionador de recursos, elaborador de estrategias de participación popular, entre otros, los que han sido medios significativos en la búsqueda del mejoramiento de los niveles de vida de la población; de ahí que sus intervenciones se pueden ejercer en áreas como la educación, salud, judicial, entre otros

En lo relacionado al ámbito educativo, según (David, 2014) la intervención del trabajador tiene como propósito velar por el bienestar del estudiante en detectar problemas familiares y sociales que repercuten en el aprovechamiento escolar, mediante la atención oportuna frente a las constantes manifestaciones de conflictos en el contexto en que se desenvuelven, siendo necesario que posea una amplia gama de conocimientos teóricos, conceptuales y metodológicos, para dar respuesta a los diversos contextos sociales, de manera especial de los sectores más vulnerables (Alava, Rodríguez, \& Párraga, 2017)

Sin embargo, este cometido, no siempre se cumple; así por ejemplo, la Universidad de la Laguna, en concordancia con la Consejería de Educación del Gobierno de Canarias, en una investigación, resalta la necesidad de incrementar en número a los trabajadores sociales en las instituciones educativas porque los problemas de absentismo, fracaso escolar, integración de inmigrantes, violencia escolar, machismo, drogodependencias, entre otras, tienen causas y consecuencias sociales donde la acción de este profesional es esencial.

Por otro lado, en relación a la acción de este profesional en la educación; en Colombia actúa dentro de un equipo interdisciplinario llamado Departamento de Bienestar Educativo, determinado por las circunstancias del momento histórico y por las concepciones de desarrollo dominantes, tales como orientar a: aumentar el porcentaje de la mano de obra utilizable, mejorar la calidad de la mano de obra disponible, aumentar el grado de tecnificación y productividad, acrecentar las posibilidades de invenciones o innovaciones favorables al desarrollo, ayudar a 
crear dirigentes aptos para resolver los problemas que trae el desarrollo, ayudar a modificar los hábitos de consumo, contribuir a la intensificación de la movilidad de la mano de obra, y favorecer la creación de una mentalidad adecuada al desarrollo para la aceptación de las modificaciones socio- culturales que lo acompañan. Aspectos que en cierta medida se contraponen al rol del trabajador social en la escuela (Gonzáles, 2003)

En el mismo país; en un estudio desarrollado, sobre el Trabajo Social y Educación, se determina que desde la perspectiva investigativa se desconocen los importantes aportes a nivel de sistematización de experiencias y de prácticas, así como también la participación en experiencias interdisciplinarias de investigación (Beltrán, 2010)

En Ecuador, un estudio desarrollado sobre la intervención del trabajador social con los niños sordos; concluyó que este profesional debe poseer competencias específicas que le permita promover procesos de cambio social y de potenciación de un ambiente armónico y efectivo de inclusión y desarrollo, mediante el trabajo multi e interdisciplinario con los diversos agentes: padres, maestros, autoridades, otros profesionales y comunidad en general (Romero, Rodríguez, \& Roldán, 2016)

Por lo descrito, se infiere que la indeterminación de las labores en el campo de la escuela en relación con el trabajo social se deriva, entre otras cosas, de la ausencia de un espacio definido desde la escuela, pero también a la ausencia de referentes claros en la formación profesional, a partir del cual surgen inquietudes sobre el papel del profesional de trabajo social en relación con los procesos educativos formales; pese a que muchas instituciones los contratan y muchos profesionales se emplean para cumplir con las labores que se les demanda en los colegios, es evidente la ausencia de discurso, de sistematización, de producción de conocimiento al respecto (Navarrete, 2016)

En concordancia a lo analizado, el artículo busca identificar la intervención del trabajador social como parte del equipo multidisciplinario del Departamento de Consejería Estudiantil (DECE) en las Unidades Educativas del Distrito 4 del cantón Portoviejo - Manabí-Ecuador, zona 13D01, que cuentan con este profesional. Para el análisis de la temática se presenta el sustento teórico sobre el Trabajo Social en la educación y sobre el Departamento de Consejería Estudiantil, como espacio de intervención del trabajador social; la metodología utilizada, los resultados obtenidos con la respectiva discusión que permitió establecer las conclusiones de la 
investigación, que aportan al mejoramiento no solo del trabajador social en particular, sino también a la educación en general.

\section{Materiales y métodos}

La investigación es de tipo cualitativa de corte transversal y descriptivo, como métodos teóricos se utilizó el histórico lógico, deductivo, análisis-síntesis y descriptivo. Como métodos empíricos se trabajó la encuesta aplicada estudiantes, docentes y directivos, la población fue de 300 estudiantes de 4 Unidades Educativas: Unidad Educativa Picoazá, Unidad Educativa Uruguay, Unidad Educativa Portoviejo y Unidad Educativa Simón Bolívar, del Cantón Portoviejo. Con un margen de error del 5\% se obtuvo una muestra de 169 estudiantes y padres de familias. El criterio de inclusión que se manejó fue relacionado con los estudiantes que han enfrentado problemáticas en el área educativa y han sido atendidos por el Departamento de Consejería Estudiantil (DECE), así como a los Trabajadores sociales, Coordinadores del Departamento y autoridades de los planteles. Y el criterio de exclusión, lo constituyen los estudiantes que no presentan dificultades y docentes tutores.

\section{Desarrollo}

\section{El trabajo social en la educación}

El Trabajo Social tiene una función de concientización, movilización y organización para que en un proceso de formación del autodesarrollo, individuos, grupos y comunidades participen activamente en la vida política, económica y social de la nación, que cree las condiciones necesarias para un nuevo modo de ser (Egg, 2017)

En lo que corresponde al Trabajo Social en el ámbito educativo y dentro de un equipo interdisciplinar, como es el Servicio de Orientación Educativa, Psicología y Profesional que aportan a la comunidad educativa, tiene su razón de ser en la necesidad de abordar la realidad en la que se interviene desde una perspectiva globalizadora, que tenga en cuenta todos los factores y elementos que interactúan en el proceso educativo, tanto en el sistema escolar como en su relación con otros sistemas e instituciones (EDUARDO, 2012)

También se la considera como la ayuda técnica que favorece el establecimiento y fortalecimiento de las relaciones entre el medio escolar, el familiar y el comunitario, la 
integración escolar y social de los niños que tienen dificultades de adaptación al contexto educativo por sus circunstancias, tal como lo manifiesta la (Federación Internacional de Trabajores Sociales, 2018), al indicar que el trabajador social se centra en la eliminación de barreras para la inclusión educativa; es decir, que su intervención en este ámbito no puede tener otro objetivo que el de contribuir a que el alumno, independientemente de su pertenencia a un entorno socio-familiar u otro, tenga las mismas oportunidades educativas que los demás, procurando que el entorno más inmediato del alumno sea lo más motivador y estimulante posible, de cara a su adaptación y rendimiento escolar, trabajando conjuntamente (NADAL, 2018)

De cara a esta intervención, para (López, 2016) es considerada indispensable en el sector educativo, pues es un espacio que construye el futuro teniendo en cuenta el presente, identifica los factores que intervienen en los procesos de reprobación y deserción escolar, realiza estudios de evaluación sobre calidad de enseñanza, realiza programas de extensión socio comunitaria que fortalezcan la educación, consolidación de escuelas para padres, capacitaciones sobre comunicación en la familia en torno al proceso educativo, orientación profesional y vocacional.

Para que el Trabajo Social cumpla con el cometido descrito, es indispensable el desarrollo de competencias personales y profesionales, tanto en su proceso formativo como día a día en el ejercicio profesional, a fin que pueda cumplir con el rol que le corresponde, que de acuerdo con (Cabrera, 2013), se circunscribe en:

a) Participar de la elaboración del Proyecto Educativo Institucional y de los Acuerdos Institucionales de Convivencia aportando elementos de fundamentación sociopedagógicos

vinculados a la Pedagogía Social, la Psicología Comunitaria y de otras Ciencias de la Educación que colaboren con los fundamentos de la Política Educativa actual.

b) Generar vínculos entre la institución educativa y la comunidad, partiendo del análisis del diagnóstico de la situación socioeducativa, institucional y comunitaria, con el aporte de elementos al análisis, estudio y construcción de criterios respecto de las oportunidades educativas, desde las perspectivas de la Pedagogía Social y del Trabajo Social, que permita la recuperación de los saberes socialmente significativos de la comunidad en un proceso de integración educativa permanente.

c) Promover el trabajo en red con otras instituciones existentes en el distrito, la región y las jurisdicciones provincial y nacional para garantizar el ingreso, la permanencia y el 
aprendizaje de los niños/as y los/las adolescentes y adultos en la escuela, con el fin de crear vínculos y espacios de corresponsabilidad, que optimicen recursos y generen propuestas

favorecedoras del aprendizaje de los estudiantes

d) Abordar desde propuestas superadoras, las problemáticas del ausentismo, el desgranamiento y el abandono escolar, reemplazando prácticas focalizadas por abordajes comunitarios que comprometan el esfuerzo de todos los miembros de la comunidad educativa.

El Departamento de Consejería Estudiantil, como espacio de intervención del trabajador social

Los Departamentos de Consejería Estudiantil (DECE) emergen como instancias fundamentales en el proceso general de formación del estudiantado. Su rol al interior de las instituciones educativas es contribuir de manera significativa al mejoramiento de las diferentes instancias que componen la experiencia educativa de niños, niñas y adolescentes, tal como lo determina (Ley Orgánica de Educación Intercultural, 2018) en su Art. 58.- Ámbito. La atención integral de los estudiantes en proceso de formación es un componente indispensable de la acción educativa y debe ser organizada e implementada por el Departamento de Consejería Estudiantil de los establecimientos educativos en todos los niveles y modalidades. Para ello, este Departamento se apoya necesariamente en la gestión de todos los miembros de la comunidad educativa. Los servicios de este organismo deben llegar a todos los estudiantes de cada establecimiento educativo.

En relación al Trabajo Social desde los DECE, es entendido como el espacio que investiga casos específicos que se refieren a las condiciones de vida de las personas, de acuerdo a sus necesidades y potencialidades, interviene también en la resolución de situaciones para mejorar el bienestar social entre las relaciones humanas e institucionales para que haya una convivencia armónica, aspectos que determinan que su accionar consiste entre otros, en detectar factores específicos que aquejan al alumno en el proceso educativo.

Este profesional se convierte en un apoyo importante, ya que junto a los otros profesionales en la prevención y promoción del bienestar integral del estudiante, considerando si el medio familiar, comunitario y social es propicio o no para su proceso de formación, actúa como mediador entre el alumno y el resto de actores educativos buscando siempre el bienestar social, 
contrarrestado factores socio culturales que afectan el desarrollo armónico de los alumnos en el entorno educativo, tales como violencia, acoso sexual, aparición de pandillas, consumo de alcohol, tabaco y otras drogas, deserción escolar, o cualquier otro que involucre la vulneración de los derechos del estudiante.

El trabajo social es tan diferente como diferente sea el entorno en el que interviene; así, en el educativo, donde se reflejan y se detectan problemáticas sociales que afectan al estudiante en todo su proceso, el área de Trabajo Social forma parte de la estrategia de intervención del DECE como una instancia mediadora entre el estudiante y el resto de actores que se vinculan al proceso educativo, buscando siempre promover su protección, la resolución de carencias emergentes o inmediatas (familia, salud, legal, social, comunitario) y la convivencia armónica; es decir, se ocupa del mejoramiento del entorno de los individuos y de atender las necesidades de una población específica, en un determinado periodo de tiempo.

Para el logro de las metas descritas, el (Ministerio de Educación, 2012), determina roles y funciones específicas:

Integrar el equipo técnico con los profesionales en psicología clínica y psicología educativa para propender al desarrollo integral de los estudiantes en todas sus áreas.

Trabajar interdisciplinariamente con todos los miembros de la comunidad educativa (especialmente con los tutores de grado o curso).

Realizar y/o ser parte de procesos de Investigación Acción Participativa de temas relevantes en el contexto educativo. Identificar factores de riesgo de los/as estudiantes y realizar estudios sociales de caso o grupo, según lo amerite, aplicando técnicas e instrumentos propios de Trabajo Social (observación, entrevistas individuales, de grupo) registrándolos a través de sus respectivos informes y/o fichas de seguimiento.

Programar, coordinar, ejecutar y evaluar acciones (talleres, conversatorios, diálogos, grupos focales, dinámicas grupales, etc.) que den respuesta a las problemáticas y necesidades de los/as actores involucrados en la Comunidad Educativa, promoviendo su corresponsabilidad a través de su participación activa. 
Conocer la problemática social, cultural, económica, familiar de los estudiantes a fin de coadyuvar en la solución de sus problemas, tratando de que estas dificultades no incidan en el rendimiento académico.

Brindar criterios técnicos necesarios para fortalecer las relaciones entre toda la comunidad educativa, preocupándose por la integración de los estudiantes al medio educativo, poniendo énfasis en niños, niñas y adolescentes que presenten dificultades de interrelaciones sociales, interviniendo oportunamente en los obstáculos que pueden impedir el desarrollo integral del estudiante.

Determinar los factores que producen absentismo, repetición, deserción y deficiente rendimiento escolar.

Contribuir a la renovación, capacitación y formación en enfoques y técnicas de innovación del Trabajo Social para la intervención socio-familiar, en el ámbito educativo.

Proponer, orientar y/o apoyar las acciones planteadas por el DECE en el ámbito socio-familiar del niño/niña o adolescente, o canalizar los casos a las distintas instancias del registro o Red de Apoyo, con el posterior seguimiento, monitoreo y evaluación.

Promover un vínculo extra institucional inmediato, en los casos en que se presenten situaciones de vulneración de derechos.

Entre las funciones propias del campo del trabajador social en su área de intervención del Departamento de Consejería Estudiantil se establece:

Función preventiva, de gran prioridad e importancia, se consideran por un lado, la estrategias de actuación precoz sobre las causas y determinantes que generan problemáticas individuales y colectivas (antes de que surjan) así como la elaboración y ejecución de estrategias de intervención en estudiantes en situación de vulnerabilidad y/o riesgo social (prevención primaria); también abarca las acciones destinadas a la detección temprana de situaciones o fenómenos puntuales y su abordaje/tratamiento oportuno (prevención secundaria); así como, las acciones de intervención y rehabilitación frente a un caso existente o ante problemáticas emergentes en la cotidianidad.

Función de atención que engloba las diferentes acciones de intervención directa y personalizada que llevan a cabo los profesionales del DECE en respuesta a las situaciones 
puntuales que vive el estudiante en el marco de su experiencia educativa, sus requerimientos o necesidades particulares. También comprende la idea de ubicar al departamento como un espacio confiable y receptivo al que puedan acudir niños, niñas y adolescentes cuando tengan cualquier tipo de inquietud, duda o problema. Esta función asimismo se basa en el establecimiento de una escucha activa.

Función de mediación, que comprende en asumir una postura que facilite la comunicación entre las personas involucradas en una situación o conflicto puntual, reduciendo la tensión existente entre las partes y actuando como un catalizador que posibilite la formulación de acuerdos y propuestas que surjan desde los mismos involucrados en beneficio de los estudiantes.

Función de seguimiento, en este sentido, es fundamental que sus profesionales lleven a cabo acciones de revisión, rastreo, indagación y monitoreo del curso y evolución de dichos procesos.

Función de capacitación e investigación, concebido como la responsabilidad de ahondar y ampliar sus conocimientos, perspectivas teóricas y técnicas para el desarrollo de su trabajo en atención a la diversidad de temáticas que surgen en la cotidianidad escolar con el estudiantado.

\section{Resultados}

Resultados obtenidos de la encuesta que tuvo como objetivo identificar la intervención del trabajador social como parte del equipo multidisciplinario del Departamento de Consejería Estudiantil. (DECE) en el Distrito 4 zona 13D01 Ecuador. Para llevar a efecto el análisis de las variables de estudio se consideró los indicadores: problemáticas detectadas y atendidas, rol e intervención del trabajador social, así como la valoración que las autoridades educativas tienen sobre el desempeño de este profesional.

\begin{tabular}{|l|l|l|l|l|l|l|}
\hline \multirow{2}{*}{ Alternativas } & \multicolumn{2}{|c|}{ Estudiantes } & \multicolumn{2}{c|}{ Trabajadores Sociales } & \multicolumn{2}{c|}{ Directivos } \\
\cline { 2 - 8 } & F & $\%$ & F & $\%$ & F & $\%$ \\
\hline De asistencia & 26 & 15.38 & 4 & 26.67 & 5 & 29.41 \\
\hline De disciplina & 73 & 43.20 & 4 & 26.67 & 5 & 29.41 \\
\hline $\begin{array}{l}\text { Rendimiento } \\
\text { académico }\end{array}$ & 64 & 37.86 & 4 & 26.67 & 5 & 29.41 \\
\hline Acoso escolar & 3 & 1.78 & 0 & 0 & 1 & 5.88 \\
\hline $\begin{array}{l}\text { Relacionados } \\
\text { con la familia }\end{array}$ & 3 & 1.78 & 3 & 20 & 1 & 5.88 \\
\hline Total. & 169 & $100 \%$ & 15 & $100 \%$ & 17 & $100 \%$ \\
\hline
\end{tabular}


Tabla 1. Principales problemáticas detectadas y atendidas en las Unidades Educativas

Fuente: Estudiantes, Trabajadores Sociales y Directivos de las Unidades Educativas

La tabla número uno muestra que las principales problemáticas detectadas y atendidas por el DECE en las Unidades Educativas investigadas, son las relacionadas con el rendimiento académico, problemas de disciplina y asistencia, resultados en los que coinciden los sujetos investigados y que perfilan en modo de actuación del trabajador social en cada uno de los contextos.

\begin{tabular}{|l|c|c|c|c|c|c|}
\hline \multirow{2}{*}{ Alternativas } & \multicolumn{2}{|c|}{ Estudiantes } & \multicolumn{2}{c|}{$\begin{array}{c}\text { Trabajadores } \\
\text { Sociales }\end{array}$} & \multicolumn{2}{c|}{ Directivos } \\
\cline { 3 - 9 } & F & \% & F & $\%$ & F & $\%$ \\
\hline a) Orientador & 84 & 49.70 & 2 & 16.67 & 1 & 7.14 \\
\hline b) Educador & 13 & 7.69 & 1 & 8.33 & 0 & 0 \\
\hline c) Capacitador & 14 & 8.28 & 4 & 33.33 & 3 & 21.43 \\
\hline d) Asistencial & 0 & 0 & 0 & 0 & 0 & 0 \\
\hline $\begin{array}{l}\text { e) Administrador de } \\
\text { proyectos }\end{array}$ & 8 & 4.73 & 1 & 8.33 & 2 & 14.29 \\
\hline f) Investigador & 48 & 28.40 & 3 & 25 & 5 & 35.71 \\
\hline $\begin{array}{l}\text { g) Evaluador de } \\
\text { servicios y proyectos }\end{array}$ & 5 & 2.95 & 1 & 8.33 & 3 & 21.43 \\
\hline $\begin{array}{l}\text { h) Organizador de } \\
\text { actividades }\end{array}$ & 3 & 1.77 & 0 & 0 & 0 & 0 \\
\hline $\begin{array}{l}\text { Total } \\
\text { Total }\end{array}$ & 9 & & 12 & $100 \%$ & 14 & $100 \%$ \\
\hline
\end{tabular}

Tabla 2. Rol del trabajador social

Fuente: Estudiantes, Trabajadores Sociales y Directivos de las Unidades Educativas

En la tabla 2 se evidencia el rol que cumple el trabajador social, visto desde cada una de las perceptivas de los sujetos investigados; así, para los estudiantes, creen que este profesional es un orientador y un investigador, respuestas que se comprenden ya que el trabajador social es quien busca la información requerida en diversos contextos: familia, compañeros, comunidad...; para los directivos el rol se enfoca como investigador, seguido de capacitador y evaluador de servicios y proyectos, enfoque percibido desde una perspectiva un poco más amplia; mientras que para los mismos trabajadores sociales el rol que cumplen con mayor frecuencia en las instituciones educativas consiste en ser capacitador seguido por investigador; como se demuestra la constante está en el rol de investigador.

\begin{tabular}{|l|c|c|c|c|c|c|}
\hline \multicolumn{1}{|c|}{ Alternativas } & \multicolumn{2}{|c|}{ Estudiantes } & \multicolumn{2}{c|}{$\begin{array}{c}\text { Trabajadores } \\
\text { Sociales }\end{array}$} & \multicolumn{2}{c|}{ Directivos } \\
\cline { 2 - 7 } & $\mathrm{F}$ & $\%$ & $\mathrm{~F}$ & $\%$ & $\mathrm{~F}$ & $\%$ \\
\hline $\begin{array}{l}\text { a) Al mejoramiento de la } \\
\text { calidad de vida de los }\end{array}$ & 89 & 52.66 & 4 & 40 & 2 & 40 \\
\hline
\end{tabular}




\begin{tabular}{|l|c|c|c|c|c|c|}
\hline $\begin{array}{l}\text { miembros de la comunidad } \\
\text { educativa }\end{array}$ & 35 & 20.71 & 4 & 40 & 1 & 20 \\
\hline $\begin{array}{l}\text { b) Al mejoramiento del } \\
\text { entorno social }\end{array}$ & 44 & 26.04 & 1 & 10 & 2 & 40 \\
\hline $\begin{array}{l}\text { c) Mejorar las relaciones } \\
\text { interpersonales }\end{array}$ & 1 & 0.59 & 1 & 10 & 0 & 0 \\
\hline $\begin{array}{l}\text { d) Respeto a los derechos de } \\
\text { los grupos vulnerables }\end{array}$ & 0 & 0 & 0 & 0 & 0 & 0 \\
\hline \begin{tabular}{l} 
e) otros Total \\
\hline
\end{tabular} & 169 & $100 \%$ & 10 & $100 \%$ & 5 & $100 \%$ \\
\hline
\end{tabular}

Tabla 3. Intervención del Trabajador Social

Fuente: Estudiantes, Trabajadores Sociales y Directivos de las Unidades Educativas

La tabla 3 expone la contribución que ha generado la intervención del trabajador social en las instituciones educativas; para los estudiantes la mayor significación ha estado en el mejoramiento de la calidad de vida de los miembros de la comunidad educativa; aspecto con el que coinciden los mismos trabajadores sociales y los directivos; estos últimos opinan que en la misma medida su acción ha contribuido al mejoramiento del entorno social de los planteles educativos., aspectos que se relacionan con la apreciación descrita anteriormente en el rol de orientador investigador que cumplen. En la misma tabla se denota que el sector menos favorecido ha sido el respeto a los derechos de los grupos vulnerables.

\begin{tabular}{|l|c|c|c|c|c|c|}
\hline \multirow{2}{*}{ Alternativas } & \multicolumn{2}{|c|}{ Estudiantes } & \multicolumn{2}{c|}{$\begin{array}{c}\text { Trabajadores } \\
\text { Sociales }\end{array}$} & \multicolumn{2}{c|}{ Directivos } \\
\cline { 2 - 7 } & F & \% & F & \% & F & $\%$ \\
\hline Excelente & 87 & 51.47 & 4 & 40 & 2 & 40 \\
\hline Buena & 55 & 32.55 & 4 & 40 & 1 & 20 \\
\hline Regular & 20 & 11.83 & 1 & 10 & 2 & 40 \\
\hline Deficiente & 7 & 4.15 & 1 & 10 & 0 & 0 \\
\hline Total & 169 & $100 \%$ & 10 & $100 \%$ & 5 & $100 \%$ \\
\hline
\end{tabular}

Tabla 4: Valoración a la acción que desarrolla el trabajador social

Fuente: Estudiantes, Trabajadores Sociales y Directivos de las Unidades Educativas

La tabla 4 muestra la apreciación valorativa que cada sector de la población investigada tiene sobre la acción que desarrolla en trabajador social; misma que de manera general va de buena a excelente; sin embargo, resulta necesario mencionar que para cierto número de estudiantes es catalogada como regular.

\section{Discusión}


En las instituciones educativas investigadas, se evidencia que los problemas que atienden los trabajadores sociales como parte del equipo multidisciplinario del DECE, son comunes; relacionados con el desempeño, disciplina, ausentismo, familia; en los que el profesional de esta área debe desarrollar su accionar; aspecto en el que se coincide con un estudio desarrollado por la Universidad Pedagógica de El Salvador, donde se determina que la educación es un área que ha venido a dar pautas de desarrollo de la sociedad, es donde puede incursionar el trabajador social, ya que no hay intervención del mismo, ante ello las alternativas de ejercer el rol de intermediador entre lo social, familiar y lo educativo, por ser áreas que determinan a este profesional, ejercer las funciones en el entorno educativo, quien integra la participación activa de los involucrados a resolver problemáticas de índole social, con el apoyo de equipo multidisciplinario, enfocados en el desarrollo social de los individuos, siendo el caso de toda la comunidad educativa, la que debe comprometerse a ser precursores del cambio (Amaya \& Mejía, 2011)

En este estudio se identificó que el rol del trabajador social es apreciado indistintamente por cada uno de los miembros de la comunidad educativa; de acuerdo a los autores de este trabajo, esto depende de la acción que el profesional realiza con cada uno de ellos; el estudiante lo percibe como orientador, para los directivos capacitador, y en el rol de investigador existe coincidencia entre los consultados; aspecto que coincide con la visión de un estudio de la Universidad de Columa, respecto a las funciones realizadas por el personal de Trabajo Social al atender los conflictos dentro de las escuelas secundarias del estado de Colima, se identifica que prevalece con mayor insistencia la de mediación, seguida de la orientación y posteriormente la de investigador (Amador, 2007)

La intervención del trabajador social en las instituciones educativas contribuye no sólo al mejoramiento del entorno social, sino también de la calidad de vida de la comunidad educativa; sin embargo, lo relacionado al respeto de los derechos de los grupos vulnerables ha sido el menos atendido; aspectos que coincide con lo expuesto por (Calvo, 2014) y (Navarrete, 2016), quienes en sus investigaciones determinan que trabajadores sociales construyen la demanda rescatando trayectorias de los sujetos, en acercamiento a la trama cotidiana, elaborando estrategias que vinculan la escuela a la realidad social y escenario particular.

Estos mismos estudios aseveran que la limitación más grande e importante para el ejercicio del trabajo social en el ámbito escolar es el propio desconocimiento acerca de la especificidad del quehacer profesional en esta área de intervención, en el que al desconocimiento y subvaloración 
de esta labor y, a la insuficiencia de recursos, se suma la inexperiencia y falta de discernimiento de los intervinientes respecto a las particularidades de la escuela y de su labor en este contexto, sobre todo con los grupos más vulnerables de la sociedad.

La acción realizada por los trabajadores sociales se reconoce como buena como parte del DECE; sin embargo, se evidencia que éstos deben ampliar su radio de acción, ya que el campo de acción es amplio y apasionante y que se extiende más allá de la presencia de equipos de orientación educativa, forman parte de las Escuelas de Padres, acompañamiento a la pareja y familia, educación social, animación socio cultural, promoción y capacitación social a autoridades, docentes y líderes de la comunidad educativo (Gonzáles \& Cívicos, s/f)

\section{Conclusiones}

La intervención del trabajador social como parte del equipo multidisciplinario del Departamento de Consejería Estudiantil (DECE) en el Distrito 4 del cantón Portoviejo Manabí-Ecuador, zona 13D01, contribuye al mejoramiento de la calidad de vida de los estudiantes, se circunscribe en la atención de casos problemas de desempeño, asistencia, atención a las familias de los estudiantes, cumpliendo funciones de orientador, capacitador e investigador; sin embargo, la atención de los derechos de los grupos vulnerables es poco atendido, aspecto que debe ser prioritario en el accionar de este profesional.

El trabajador social interviene ante la presencia de situaciones que alteran la armonía del desarrollo formativo de los estudiantes; sin embargo, es necesario que promueva estrategias de actuación precoz, o llamadas también de prevención tanto primaria como secundaria, en situación de vulnerabilidad y/o riesgo social; así como la detección temprana de situaciones o fenómenos puntuales y su abordaje oportuno ante problemáticas emergentes en la cotidianidad.

\section{Referencias Bibliográficas}

Alava, L., Rodríguez, A., \& Párraga, F. (2017). El perfil profesional del trabajador social del siglo XXI. Revista Caribeña de Ciencias Sociales, En línea: http://www.eumed.net/rev/caribe/2017/04/trabajador-social-pefil.html .

Amador, J. (2007). La intervención del trabajador social en los conflictos escolares que se presentan en el nivel secundario. Colima: Universidad de Columa. 
Amaya, C., \& Mejía, M. (2011). El trabajador social en la institución educativa, caso de 5 centros escoalres, San Salvador, 2010-2011. San Salvador: Universidad Pedagógica.

Beltrán, J. (2010). Trabajo social y educación: reflexiones en torno al debate formativo y profesional contemporáneo. Bogotá: Universidad Nacional de Colombia.

Calvo, M. (2014). La intervención del Trabajo Social en el campo educativo: enfoque de derechos. Argentina: Universidad Nacional de la Plata.

Cárdenas, H. (2014, Noviembre 11). TRABAJO SOCIAL, SU HISTORIA, PROCESO, DESARROLLO Y FUNCIONES. Obtenido de Historia y Antecedente del Trabajo Social: http://hildacardenas2014.blogspot.com/2014/11/el-trabajo-social-su-historiaprocesos.html

Egg, E. A. (2017, Noviembre 24). MI TRABAJO ES SOCIAL. Obtenido de Historia del Trabajo Social :

http://webcache.googleusercontent.com/search?q=cache:http://www.mitrabajoessocial .com/historia-del-trabajo-socialezequiel-ander-egg/

Frank, H. (2018, 02 28). Trabajo Social. Obtenido de Trabajo social en Latinoamerica: http://hablandodetrabajosocial.blogspot.com/2009/11/trabajo-social-enlatinoamerica.html

Gonzáles, A., \& Cívicos, A. (s/f). Análisis de funciones del trabajador social en el campo educativo. Islas Canarias: Universidad de la Laguna.

Hernández, M. (2013). Análisis de funciones del Trabajador Social en el campo educativo. Londres: S\N.

LEY ORGÁNICA DE EDUCACIÓN INTERCULTURAL. (2018, Marzo 14). MINISTERIO DE EDUCACIÓN. Obtenido de https://educacion.gob.ec/wpcontent/uploads/downloads/2017/02/Reglamento-General-a-la-Ley-OrgAnica-deEducacion-Intercultural.pdf

Loor, M. J. (2016). el trabajo social. Itsup, 32-34.

MINISTERIO DE EDUCACIÓN. (2012, Julio). DIRECCIÓN NACIONAL DE EDUCACIÓN PARA LA DEMOCRACIA Y EL BUEN VIVIR. Obtenido de MODELO DE 
Rev. SINAPSIS, Edición № 12, Vol. 1, Diciembre 2018

ISSN 1390 - 9770

ATENCIÓN INTEGRAL DE LOS DEPARTAMENTO DE CONSEJERIA

ESTUDIANTIL: https://educacion.gob.ec/wp-

content/uploads/downloads/2015/03/modelo_atenci\%C3\%B3n_integral_departamento s_de_consejer\%C3\%ADa_estudiantil_-_dece.pdf

Navarrete, N. (2016). El papel del trabajo social en el ámbito eductaivo. Folios de Humanidades, 1-10.

OMG. (2018, febrero 24). Salud de vida. Obtenido de www.omgeslamejor

Romero, S., Rodríguez, A., \& Roldán, N. (2016). El trabajador social y las competencias para la intervención social y familiar con niños sordos. Rev. SINAPSIS, 2(9). 